\title{
Optical Wireless Links with Spatial Diversity over Strong Atmospheric Turbulence Channels
}

\author{
Theodoros A. Tsiftsis, Member, IEEE, Harilaos G. Sandalidis, George K. Karagiannidis, Senior Member, IEEE, \\ and Murat Uysal, Senior Member, IEEE
}

\begin{abstract}
Optical wireless, also known as free-space optics, has received much attention in recent years as a cost-effective, licensefree and wide-bandwidth access technique for high data rates applications. The performance of free-space optical (FSO) communication, however, severely suffers from turbulence-induced fading caused by atmospheric conditions. Multiple laser transmitters and/or receivers can be placed at both ends to mitigate the turbulence fading and exploit the advantages of spatial diversity. Spatial diversity is particularly crucial for strong turbulence channels in which single-input single-output (SISO) link performs extremely poor. Atmospheric-induced strong turbulence fading in outdoor FSO systems can be modeled as a multiplicative random process which follows the $\mathrm{K}$ distribution. In this paper, we investigate the error rate performance of FSO systems for K-distributed atmospheric turbulence channels and discuss potential advantages of spatial diversity deployments at the transmitter and/or receiver. We further present efficient approximated closed-form expressions for the average bit-error rate (BER) of single-input multiple-output (SIMO) FSO systems. These analytical tools are reliable alternatives to time-consuming Monte Carlo simulation of FSO systems where BER targets as low as $10^{-9}$ are typically aimed to achieve.
\end{abstract}

Index Terms-Atmospheric turbulence, bit-error rate (BER), free-space optical communication, $K$ distribution, optical wireless, spatial diversity.

\section{INTRODUCTION}

$\mathbf{F}$ REE-SPACE OPTICAL (FSO) communication is a license-free and cost-effective access technique, which has attracted significant attention recently for a variety of applications [1], [2]. Channels in FSO systems have wider bandwidth and therefore are able to support more users compared to radio frequency (RF) counterparts. Through relaying techniques, outdoor FSO optical transceivers can also cover large distances [3], [4]. With its high-data-rate capacity and wide bandwidth on unregulated spectrum, FSO communication is a promising solution for the "last mile" problem,

Manuscript received November 25, 2007; revised March 19, 2008; accepted May 19, 2008. The associate editor coordinating the review of this paper and approving it for publication was G. Durgin.

This paper was presented in part at the IEEE International Conference on Communications (ICC'08), Beijing, China, May 2008.

T. A. Tsiftsis and G. K. Karagiannidis are with the Department of Electrical and Computer Engineering, Aristotle University of Thessaloniki, 54124 Thessaloniki, Greece (e-mail: \{thtsif, geokarag\} @auth.gr).

H. G. Sandalidis is with the Department of Informatics with Applications to Biomedicine, University of Central Greece, Papassiopoulou 2-4, 35100 Lamia, Greece (e-mail: sandalidis@hotmail.com).

M. Uysal is with the Department of Electrical and Computer Engineering, University of Waterloo, Waterloo, Ontario, Canada, N2L 3G1 (e-mail: muysal@ece.waterloo.ca).

Digital Object Identifier 10.1109/TWC.2009.071318 however its performance is highly vulnerable to adverse atmospheric conditions. Atmospheric turbulence occurs as a result of the variations in the refractive index due to inhomogeneities in temperature and pressure changes. This results in rapid fluctuations at the received signal, i.e. known as fading or scintillation, impairing the system performance particulary for link ranges for $1 \mathrm{~km}$ and above.

Over the years, a number of statistical channel models have been proposed to describe weak or strong atmospheric-induced turbulence fading [1]. For strong turbulence conditions, the $\mathrm{K}$ distribution has been found to be a suitable model since it provides an excellent agreement between theoretical and experimental data [5]. In [6], Uysal and Li have used this channel model to evaluate the performance of coded FSO systems in terms of the pairwise error probability and biterror rate (BER). In [7], they have extended their results for a correlated $\mathrm{K}$ turbulence model where an exponential correlation profile is adopted. In [8], Kiasaleh has studied the BER performance of a FSO heterodyne system over the $\mathrm{K}$ channel. The results in these papers demonstrate that the performance of single-input single-output (SISO) FSO links severely suffers from strong turbulence and is far away from satisfying the typical BER targets for FSO applications within the practical ranges of signal-to-noise ratio. This necessitates the deployment of powerful fading-mitigation techniques. In the existing literature on FSO communication, two techniques have been proposed to mitigate the degrading effects of atmospheric turbulence: Error control coding in conjunction with interleaving [7], [9] and maximum likelihood sequence detection (MLSD) [10]. However, both approaches come with some practical limitations. The first one requires large-size interleavers whereas the later suffers from high computational complexity.

Another promising solution is the use of spatial diversity, a well known diversity technique in RF systems. By using multiple apertures at the transmitter and/or the receiver, the inherent redundancy of spatial diversity has the potential to significantly enhance the performance. The possibility for temporal blockage of the laser beams by obstructions is further reduced and longer distances can be covered through heavier weather conditions. The use of space diversity in FSO systems has been first proposed in [11]. In [12], [13], Shin and Chan have investigated the outage probability of multiple-input multiple-output (MIMO) FSO systems over log-normal turbulence channels. In [14], [15] Wilson et. al have studied MIMO FSO transmissions assuming pulseposition-modulation (PPM) [14] and Q-ary PPM [15] both in 
log-normal and Rayleigh fading regimes. In [16], Navidpour et.al. have studied the BER performance of MIMO FSO links for both independent and correlated log-normal atmospheric turbulence channels.

In this paper, we investigate the performance of MIMO FSO links over independent and not necessarily identically distributed (i.n.i.d.) $\mathrm{K}$ turbulence channels. We assume intensity modulation/direct-detection (IM/DD) with on-off keying (OOK). First, as a benchmark, we derive a closed-form expression for the BER of SISO case. Then, we present highly accurate approximated closed-form BER expressions for FSO links with multiple apertures at the receiver. All the derived expressions are given in terms of the well-known Meijer G-functions which are available as built-in functions of many commercial mathematical software packages. These expressions are highly efficient analytical tools and stand out as reliable alternatives to time-consuming Monte Carlo simulation of FSO systems where very low BER targets (from $10^{-6}$ to $10^{-9}$ ) are aimed to achieve.

The remainder of the paper is organized as follows. In Section II, the system model is introduced and the K distribution atmospheric turbulence is described. In Section III, we present a closed-form BER expression for SISO FSO links while in Section IV multiple transmit or receive apertures deployments are investigated for optimal combining ${ }^{1}$ (OC), equal-gain combining (EGC) and selection combining (SC) diversity receivers. In Section $\mathrm{V}$, a number of numerical examples are presented to confirm the accuracy of the derived expressions and the advantages of using spatial diversity on the FSO links are discussed. Finally, useful concluding remarks are provided in Section VI.

\section{System And Channel Model}

\section{A. System Model}

An FSO system is considered where the information signal is transmitted via $M$ apertures and received by $N$ apertures over a discrete-time ergodic channel with additive white Gaussian noise (AWGN). We assume binary-input and continuous output and IM/DD with OOK. The received signal at the $n$th receive aperture is given by

$$
r_{n}=x \eta \sum_{m=1}^{M} I_{m n}+v_{n}, n=1, \ldots, N
$$

where $x \in\{0,1\}$ represents the information bits, $\eta$ is the optical-to-electrical conversion coefficient, $I_{m n}$ denotes the irradiance from the $m$ th transmitter to the $n$th receiver, and $v_{n}$ is the AWGN with zero mean and variance $\sigma_{v}=N_{0} / 2$. Under the Gaussian noise approximation, it has been implicitly assumed that the presence of ambient light in photodetectors can be ignored. Although it is a major source of interference particularly during daylight, it can be significantly reduced using infrared filters over the photodiodes in practical FSO implementations. Considering that the coherence length of the optical beams is of the order of centimeters, the channel

\footnotetext{
${ }^{1}$ In this paper we use the term OC instead of maximal-ratio combining (MRC) which is common in the wireless communications literature. However, there are papers in optics literature where the term MRC is used, e.g. [17], [18].
}

fades can be assumed as independent if the transmitters and/or receivers are placed a few centimeters apart.

\section{B. Channel Statistics}

Strong atmospheric turbulence is modeled using a widely accepted distribution, the $\mathrm{K}$ distribution [5]. $\mathrm{K}$ turbulence model can be considered as a product of two independent models [7], (i.e., exponential distribution $*$ gamma distribution) and its probability density function (pdf) of the normalized irradiance is given by

$$
\begin{aligned}
& f_{I_{m n}}\left(I_{m n}\right) \\
& =\frac{2 \alpha_{m n}^{\frac{\left(\alpha_{m n}+1\right)}{m n^{2}}}}{\Gamma\left(\alpha_{m n}\right)} I_{m n^{2}}^{\frac{\left(\alpha_{m n-1}\right.}{2}} K_{\alpha_{m n}-1}\left(2 \sqrt{\alpha_{m n} I_{m n}}\right), I_{m n}>0
\end{aligned}
$$

where $\alpha_{m n}$ is a channel parameter related to the effective number of discrete scatterers, $\Gamma(\cdot)$ is the Gamma function [19, eq. (8.310.1)], and $K_{\nu}(\cdot)$ is the $\nu$ th-order modified Bessel function of the second kind [19, eq. (8.432.2)]. When $\alpha_{m n} \rightarrow$ $\infty,(2)$ approaches the negative exponential (NE) distribution. By writing the $K_{\nu}(\cdot)$ in terms of the Meijer G-function given in $[20$, eq. $(8.4 .23 .1)]$ as

$$
K_{\nu}(x)=\frac{1}{2} G_{0,2}^{2,0}\left[x^{2} /\left.4\right|_{\frac{\nu}{2},-\frac{\nu}{2}}-{ }^{-},\right.
$$

the cumulative distribution function (cdf) of $I$ can be easily derived from (2) with the help of [20, eq. (2.24.2.2)] as

$$
F_{I_{m n}}\left(I_{m n}\right)=\frac{1}{\Gamma\left(\alpha_{m n}\right)} G_{1,3}^{2,1}\left[\begin{array}{l|c}
\alpha_{m n} I_{m n} & 1 \\
\alpha_{m n}, 1,0
\end{array}\right] \text {. }
$$

Note that Meijer G-function [19, eq. (9.30)] is a standard built-in function in most of the well-known mathematical software packages such as Mathematica and Maple. Additionally, using [19, eq. (9.303)], the Meijer G-function can be written in terms of the more familiar generalized hypergeometric functions [19, eq. (9.14.1)].

The $n$-th order moment represented by $\mu_{I_{m n}}(n)=\int_{0}^{\infty} I_{m n}^{n} f_{I_{m n}}\left(I_{m n}\right) d I_{m n}$ is given in a closed form using [21, eq. (24)] as

$$
\mu_{I_{m n}}(n)=\frac{\Gamma(n+1) \Gamma\left(n+\alpha_{m n}\right)}{\alpha_{m n}^{n} \Gamma\left(\alpha_{m n}\right)} .
$$

Using (5) we can calculate the scintillation index (SI) as

$$
S I \triangleq \frac{E\left[I_{m n}^{2}\right]-E^{2}\left[I_{m n}\right]}{E^{2}\left[I_{m n}\right]}=\frac{\alpha_{m n}+2}{\alpha_{m n}}
$$

where $E[\cdot]$ denotes the expected value of the enclosed. Since $S I$ depends only on the parameter $\alpha_{m n}$, one can see that the turbulence is stronger ( $S I$ is high) for lower values of $\alpha_{m n}$ and gets weaker as $\alpha_{m n}$ increases.

\section{Electrical SNR Statistics}

The instantaneous electrical signal-to-noise ratio (SNR) can be defined as $\gamma_{m n}=\left(\eta I_{m n}\right)^{2} / N_{0}$. The average electrical $\mathrm{SNR}$ is defined as, ${ }^{2} \mu_{m n}=\left(\eta E\left[I_{m n}\right]\right)^{2} / N_{0}$ [22]. After a

\footnotetext{
${ }^{2}$ Note that $E[I]=1$ since $I_{m n}$ is normalized. Also $\mu$ is different than $\bar{\gamma}=E[\gamma]$ since the latter quantity is defined as $\bar{\gamma}=\eta^{2} E\left[I^{2}\right] / N_{0}$.
} 
simple power transformation of the random variable (rv) $I_{m n}$, the pdf of the electrical SNR, $\gamma_{m n}$, can be derived as

$$
\begin{aligned}
& f_{\gamma_{m n}}\left(\gamma_{m n}\right) \\
& =\frac{\alpha_{m n^{2}}^{\frac{\alpha_{m n}}{2}} \gamma_{m n^{4}}^{\frac{\alpha_{m n-3}}{\alpha^{4}}}}{\Gamma\left(\alpha_{m n}\right) \mu_{m n^{4}}^{\frac{\alpha_{m n+1}}{m n^{4}}}} K_{\alpha_{m n}-1}\left(2 \sqrt{\alpha_{m n} \sqrt{\frac{\gamma_{m n}}{\mu_{m n}}}}\right), \gamma_{m n}>0 .
\end{aligned}
$$

The cumulative distribution function (CDF) of $\gamma_{m n}$ is then

$$
F_{\gamma_{m n}}\left(\gamma_{m n}\right)=\frac{1}{\Gamma\left(\alpha_{m n}\right)} G_{1,3}^{2,1}\left[\alpha_{m n} \sqrt{\frac{\gamma_{m n}}{\mu_{m n}}} \mid \begin{array}{c}
1 \\
\alpha_{m n}, 1,0
\end{array}\right]
$$

\section{SISO FSO LINKS}

The BER of IM/DD with OOK in the presence of AWGN and perfect CSI at the receiver side is given by $P_{e}=$ $P(1) P(e \mid 1)+P(0) P(e \mid 0)$ where $P(1)$ and $P(0)$ are the probabilities of sending 1 and 0 bits, respectively, and $P(e \mid 1)$ and $P(e \mid 0)$ denote the conditional bit-error probabilities when the transmitted bit is 1 and 0 . Due to the symmetry of the problem, we consider that $P(1)=P(0)=0.5$ and $P(e \mid 1)=P(e \mid 0)$. It is easy to show that conditioned on $I$ (the indexes $m, n$ are omitted for brevity), we have [16]

$$
P(e \mid I)=P(e \mid 1, I)=P(e \mid 0, I)=Q\left(\frac{\eta I}{\sqrt{2 N_{0}}}\right)
$$

where $Q(\cdot)$ is the Gaussian $Q$-function defined as $Q(x)=(1 / \sqrt{2 \pi}) \int_{x}^{\infty} \exp \left(-t^{2} / 2\right) d t$ and also related to the complementary error function $\operatorname{erfc}(\cdot)$ by $\operatorname{erfc}(x)=2 Q(\sqrt{2} x)$.

The average BER, $P_{b}(e)$, over the $\mathrm{K}$ channel can be obtained by averaging (9) over the fading coefficient $I$, i.e.,

$$
P_{e}=\int_{0}^{\infty} f_{I}(I)\left[\frac{1}{2} \operatorname{erfc}\left(\frac{\eta I}{2 \sqrt{N_{0}}}\right)\right] d I .
$$

The above integral can be evaluated by expressing the $K_{\nu}(\cdot)$ and the $\operatorname{erfc}(\cdot)$ integrands as Meijer G-functions $(\operatorname{erfc}(\sqrt{x})=$ $\frac{1}{\sqrt{\pi}} G_{1,2}^{2,0}\left[\left.x\right|_{0,1 / 2} ^{1}\right]$ [20, eq. (8.4.14.2)]) and using [21, eq. (21)]. Therefore, a closed-form solution yields as

$$
P_{e, S I S O}=\frac{2^{\alpha-2}}{\sqrt{\pi^{3}} \Gamma(\alpha)} G_{5,2}^{2,4}\left[\frac{4 \eta^{2}}{N_{0} \alpha^{2}} \mid \begin{array}{c}
\frac{1-\alpha}{2}, \frac{2-\alpha}{2}, 0, \frac{1}{2}, 1 \\
0, \frac{1}{2}
\end{array}\right] .
$$

Alternatively, if we express (9) in terms of $\gamma$, i.e., $Q\left(\frac{n I}{\sqrt{2 N_{\mathrm{O}}}}\right)=Q\left(\sqrt{\frac{\gamma}{2}}\right)=\frac{1}{2} \operatorname{erfc}\left(\frac{\sqrt{\gamma}}{2}\right)$, and average over the pdf of $\gamma$, the above average BER can be expressed as

$$
P_{e, S I S O}=\frac{2^{\alpha-2}}{\sqrt{\pi^{3}} \Gamma(\alpha)} G_{5,2}^{2,4}\left[\frac{4 \mu}{\alpha^{2}} \mid \begin{array}{c}
\frac{1-\alpha}{2}, \frac{2-\alpha}{2}, 0, \frac{1}{2}, 1 \\
0, \frac{1}{2}
\end{array}\right] .
$$

\section{MIMO FSO LINKS}

Since the BER performance of SISO FSO link is quite poor (i.e., higher than $10^{-3}$ in the SNR range of $30-50 \mathrm{~dB}$ as it will be later demonstrated through our numerical results) over strong turbulence, the use of diversity techniques is absolutely necessary. The use of spatial diversity can be implemented either at the transmitter (MISO) or at the receiver (SIMO) or at both of them (MIMO). The optimum decision metric for OOK is given by [16, eq. (16)]

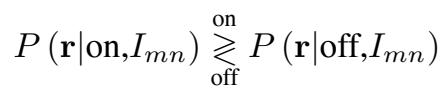

where $\mathbf{r}=\left(r_{1}, r_{2}, \ldots r_{n}\right)$ is the received signal vector. By following a similar analysis as in [16] for the conditional probabilities of the received vector being in "on" or in "off" state, the average error rate can be calculated from

$$
\begin{aligned}
& P_{e, M I M O} \\
& =\int_{\mathbf{I}} f_{\mathbf{I}}(\mathbf{I}) Q\left(\frac{\eta}{M N \sqrt{2 N_{0}}} \sqrt{\sum_{n=1}^{N}\left(\sum_{m=1}^{M} I_{m n}\right)^{2}}\right) d \mathbf{I}
\end{aligned}
$$

where $f_{\mathbf{I}}(\mathbf{I})$ is the joint pdf of vector $\mathbf{I}=\left(I_{11}, I_{12}, \ldots I_{M N}\right)$ of length $M N$. The average BER in (14) can be calculated through multi-dimensional numerical integration and with the help of mathematical software packages. In order to fairly compare MIMO links with SISO one, the factor $M$ is used in (14) to ensure that the total transmit power of the MISO FSO system is the same as the power of the SISO link. Moreover, the factor $N$ ensures that the area of the receive aperture in SISO links has the same size with the sum of $N$ receive aperture areas of SIMO links [12]. To have further insight into the performance of FSO links with spatial diversity, we investigate the transmit and receive diversity as special cases.

\section{A. MISO FSO Links}

When transmit diversity is used, i.e., $N=1$, (14) is written as

$$
P_{e, M I S O}=\int_{\mathbf{I}} f_{\mathbf{I}}(\mathbf{I}) Q\left(\frac{\eta}{M \sqrt{2 N_{0}}} \sum_{m=1}^{M} I_{m}\right) d \mathbf{I}
$$

which requires $M$-dimensional integration. Specifically, the multidimensional Gaussian quadrature rule (GQR) can be efficiently applied, since it involves multiple averaging of a Gaussian Q-function over the joint pdf vector $f_{\mathbf{I}}(\mathbf{I})$. The calculation of GQR provides a set of weights and abscissas [23, eq. (25.4.45); p.923, table (25.9)] such that the approximation

$$
\int_{a}^{b} G(x) W(x) d x \approx \sum_{j=1}^{K} w_{j} G\left(x_{j}\right)
$$

is exact if $G(X)$ is a polynomial of degree up to $2 K-1$ [24]. The values of $w_{j}$ and $x_{j}$ depend on the weight function and the integration interval, and can be computed by finding a set of orthogonal polynomials over $W(x)$ on $[a, b]$. From the algorithm proposed in [25], if $W(x)$ is the joint pdf of the rvs $I_{m}$, the $K$-point GQR can be computed using the first $2 K-1$ moments of $I_{m}$, which are derived in closed-form in (5).

\section{B. SIMO FSO Links}

1) Optimal Combining: When receive diversity is applied, the variance of the noise in each aperture is $N$ times smaller since the variance of the noise in each receiver is $\sigma_{v}^{2}=\frac{N_{0}}{2 N}$. 
Therefore, for $M=1$ and $\mathrm{OC}$ implementation at the receiver with perfect CSI, (14) is written as

$$
P_{e, O C}=\int_{\mathbf{I}} f_{\mathbf{I}}(\mathbf{I}) Q\left(\frac{\eta}{\sqrt{2 N N_{0}}} \sqrt{\sum_{n=1}^{N} I_{n}^{2}}\right) d \mathbf{I} .
$$

The integral presented in (17) is difficult if not impossible, to be evaluated in closed form. For that reason we use the approximation for the $Q$-function presented in [26, eq. (14)] (i.e., $Q(x) \approx \frac{1}{12} \mathrm{e}^{-\frac{x^{2}}{2}}+\frac{1}{4} \mathrm{e}^{-\frac{2 x^{2}}{3}}$ ) and thus the average BER can be evaluated as

$$
\begin{aligned}
P_{e, O C} \approx & \frac{1}{12} \prod_{n=1}^{N} \int_{0}^{\infty} f_{I_{n}}\left(I_{n}\right) e^{-\frac{\eta^{2}}{4 N N_{0}} I_{n}^{2}} d I_{n} \\
& +\frac{1}{4} \prod_{n=1}^{N} \int_{0}^{\infty} f_{I_{n}}\left(I_{n}\right) e^{-\frac{\eta^{2}}{3 N N_{0}} I_{n}^{2}} d I_{n}
\end{aligned}
$$

By applying (2), (3) in (18) and introduce also the exponential function in terms of the Meijer G-function presented in [21, eq. (11)] as

$$
e^{-x}=G_{0,1}^{1,0}\left[\left.x\right|_{0} ^{-}\right],
$$

the error rate of the $\mathrm{OC}$ diversity receiver can be evaluated using [20, eq. (2.24.4.3)] as

$$
\begin{aligned}
P_{e, O C} & \approx \frac{1}{12} \prod_{n=1}^{N} \frac{2^{\alpha_{n}-1}}{\pi \Gamma\left(\alpha_{n}\right)} G_{4,1}^{1,4}\left[\frac{4 \eta^{2}}{\alpha_{n}^{2} N N_{0}} \mid \frac{1-\alpha_{n}}{2}, \frac{2-\alpha_{n}}{0^{2}}, 0, \frac{1}{2}\right] \\
& +\frac{1}{4} \prod_{n=1}^{N} \frac{2^{\alpha_{n}-1}}{\pi \Gamma\left(\alpha_{n}\right)} G_{4,1}^{1,4}\left[\frac{16 \eta^{2}}{3 \alpha_{n}^{2} N N_{0}} \mid \frac{1-\alpha_{n}}{2}, \frac{2-\alpha_{n}}{0}, 0, \frac{1}{2}\right] .
\end{aligned}
$$

Equation (20) can be rewritten also in terms of the average electrical SNR as

$$
\begin{aligned}
P_{e, O C} & \approx \frac{1}{12} \prod_{n=1}^{N} \frac{2^{\alpha_{n}-1}}{\pi \Gamma\left(\alpha_{n}\right)} G_{4,1}^{1,4}\left[\frac{4 \mu_{n}}{\alpha_{n}^{2} N} \mid \frac{1-\alpha_{n}}{2}, \frac{2-\alpha_{n}}{2}, 0, \frac{1}{2}\right] \\
& +\frac{1}{4} \prod_{n=1}^{N} \frac{2^{\alpha_{n}-1}}{\pi \Gamma\left(\alpha_{n}\right)} G_{4,1}^{1,4}\left[\frac{16 \mu_{n}}{3 \alpha_{n}^{2} N} \mid \frac{1-\alpha_{n}}{2}, \frac{2-\alpha_{n}}{2}, 0, \frac{1}{2}\right] .
\end{aligned}
$$

where $\alpha_{n}$ is the $n$th channel parameter and $\mu_{n}$ is the average electrical SNR at the output of the $n$th diversity aperture.

2) Equal gain Combining (EGC): For the case where EGC is implemented at the receiver side (i.e., the receiver adds the receiver branches) the average error rate can be expressed as

$$
P_{e, E G C}=\int_{\mathbf{I}} f_{\mathbf{I}}(\mathbf{I}) Q\left(\frac{\eta}{N \sqrt{2 N_{0}}} \sum_{n=1}^{N} I_{n}\right) d \mathbf{I} .
$$

It should be emphasized here, that the resulting expression is equivalent to the one obtained for the MISO FSO links given by (15) assuming EGC at the receiver side. Also, it is interesting to note that although EGC is used at the receiver, the knowledge for CSI is still needed for threshold calculation on the decision rule as discussed in [16, eqs. (31) and (32)].

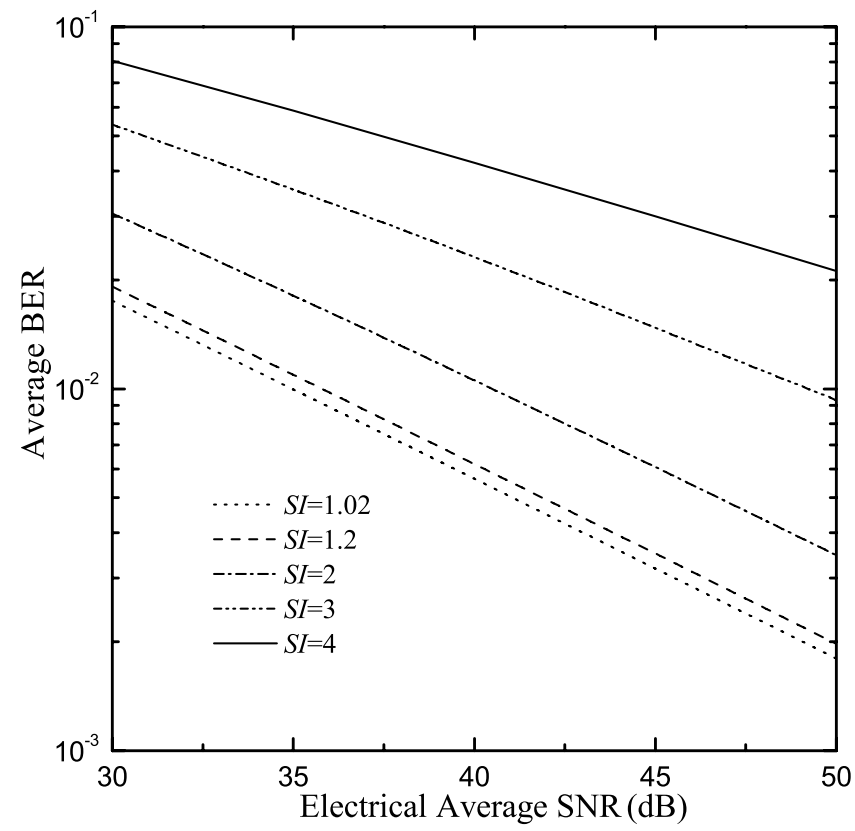

Fig. 1. Average BER of SISO FSO links as a function of $S I$.

3) Selection Combining (SC): Among the considered combining schemes, the SC is the least complicated since it processes only one of the diversity apertures and specifically the aperture with the maximum received irradiance (or electrical SNR). Therefore, the selection is made according to

$$
I_{S C}=\max \left(I_{1}, I_{2}, \ldots, I_{n}\right) .
$$

The average BER at the output of SC receiver can be expressed as

$$
P_{e, S C}=\int_{0}^{\infty} f_{I_{S C}}\left(I_{S C}\right) Q\left(\frac{\eta I_{S C}}{\sqrt{2 N N_{0}}}\right) d I_{S C}
$$

where $f_{I_{S C}}\left(I_{S C}\right)$ is the pdf of the output which can be evaluated as

$$
\begin{aligned}
f_{I_{S C}}\left(I_{S C}\right) & =\frac{d}{d I_{S C}} F_{I_{S C}}\left(I_{S C}\right)=\frac{d}{d I_{S C}} \prod_{j=1}^{N} F_{I_{j}}\left(I_{S C}\right) \\
& =\sum_{i=1}^{N} \prod_{j=1, j \neq i}^{N} f_{I_{i}}\left(I_{S C}\right) F_{I_{j}}\left(I_{S C}\right) .
\end{aligned}
$$

By applying (25) in (24) the average error can be calculated via the sum of $N$ semi-infinite integrals

$$
\begin{aligned}
P_{e, S C}=\sum_{i=1}^{N} & \prod_{j=1, j \neq i}^{N} \int_{0}^{\infty} f_{I_{i}}\left(I_{S C}\right) \\
& \times F_{I_{j}}\left(I_{S C}\right) Q\left(\frac{\eta I_{S C}}{\sqrt{2 N N_{0}}}\right) d I_{S C} .
\end{aligned}
$$

The integral in (26) can be also evaluated by GQR as presented above for the MISO case.

\section{NUMERICAL EXAMPLES \& DisCUSSION}

In this section, the error performance of MISO and SIMO deployment of apertures is investigated. 


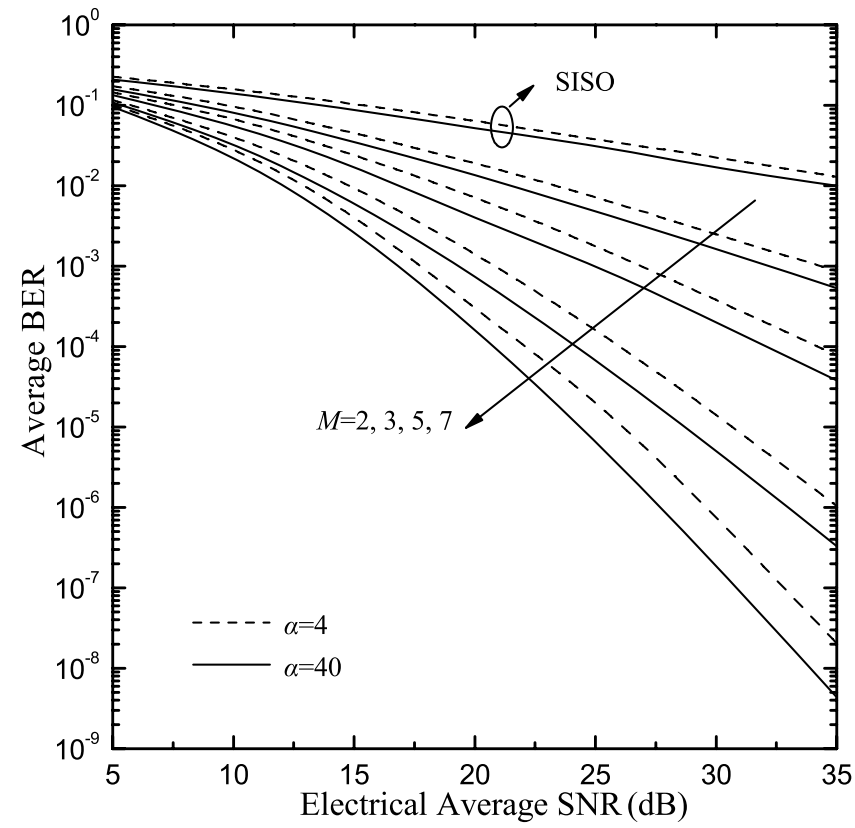

Fig. 2. Comparison of the average BER between SISO and MISO FSO links assuming perfect CSI over i.i.d. turbulent channels

In Fig. 1, the average BER in terms of $\mu$ for various parameters of the scintillation index, is depicted. We particularly examine the performance when $S I$ takes values between 1 and 4. Note that the $S I$ in (6) is invalid for $S I \leq 1$. We observe that as $S I$ increases, the turbulence effect is getting stronger and thus the BER increases. This is expected since $\alpha$ decreases as it is inversely proportional to $S I$. In the limiting case of $S I=1, \alpha \rightarrow \infty$ and hence a low BER bound exists. It is obvious that even for high values of average electrical SNR (i.e, 30-50 dB) BER is not exceeding $10^{-3} \mathrm{~dB}$ which is not an acceptable BER for practical FSO systems. This fully justifies the use of spatial diversity.

In Fig. 2 the average BER performance of MISO FSO links with $M=2,3,5$, and 7 transmit apertures over independent and identically distributed (i.i.d.) atmospheric turbulence channels with $\alpha=4$ or $\alpha=40$, is depicted. It is clearly depicted that the average BER is significantly improved as the number of transmit antennas increases compared to the SISO deployment which is also depicted. Indeed, it can be easily derived that with $M=3$ transmit apertures and $\alpha=40$ it can be obtained an SNR improvement of about $110 \mathrm{~dB}$ with respect to SISO at a target $\mathrm{BER}=10^{-9}$.

In Fig. 3 the error performance of SIMO FSO links with $N=2,3$ receive apertures employing EGC and OC over i.n.i.d. atmospheric turbulence channels, is illustrated. It is shown that the performance of EGC receivers is close to OC receivers. Specifically, for $N=2$ there is only a $1.2 \mathrm{~dB}$ difference at $\mathrm{BER}=10^{-9}$. The difference in the performance between EGC and OC receivers is expected to be similar for more receive apertures, as also presented in [16] for weak turbulence. However, it is not plotted here since the results are difficult if not impossible to be extracted for EGC. This result (i.e., similar error performance of EGC and OC receive apertures) demonstrates the aperture averaging effect i.e., a number of small receive apertures provide a similar

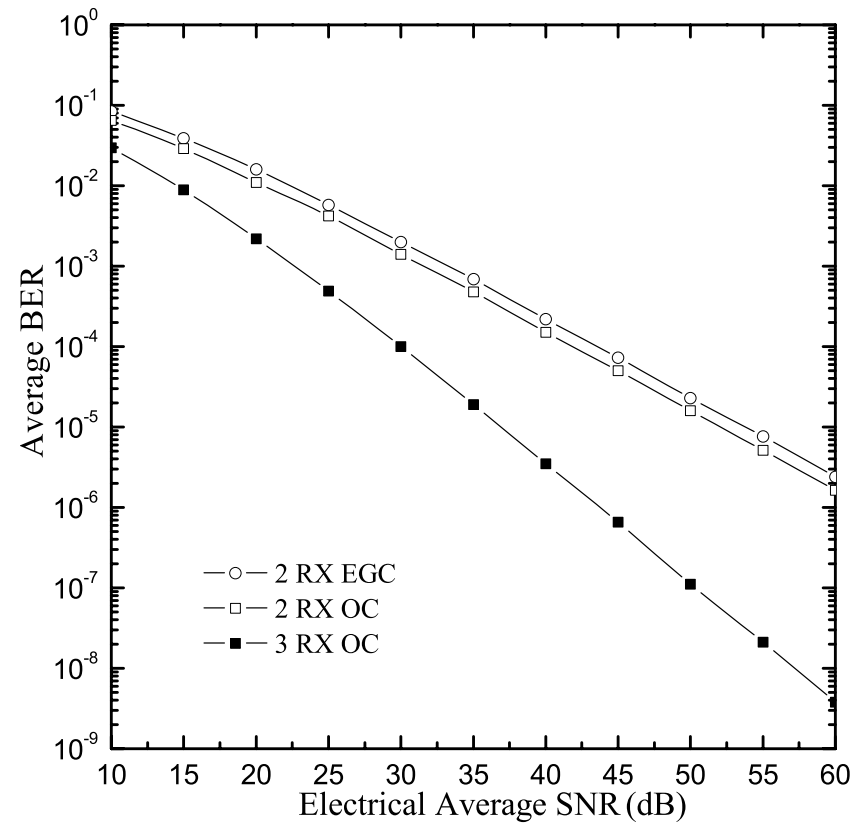

Fig. 3. Comparison of the OC and EGC receivers SIMO FSO links over i.n.i.d. atmospheric turbulence channels for $N=2\left(\alpha_{1}=4, \alpha 2=40, \mu_{1}=\right.$ $\left.\mu, \mu_{2}=2 \mu\right)$ and $N=3\left(\alpha_{1}=4, \alpha 2=10, \alpha_{3}=50, \mu_{1}=\mu, \mu_{2}=\right.$ $\left.2 \mu, \mu_{3}=4 \mu\right)$.

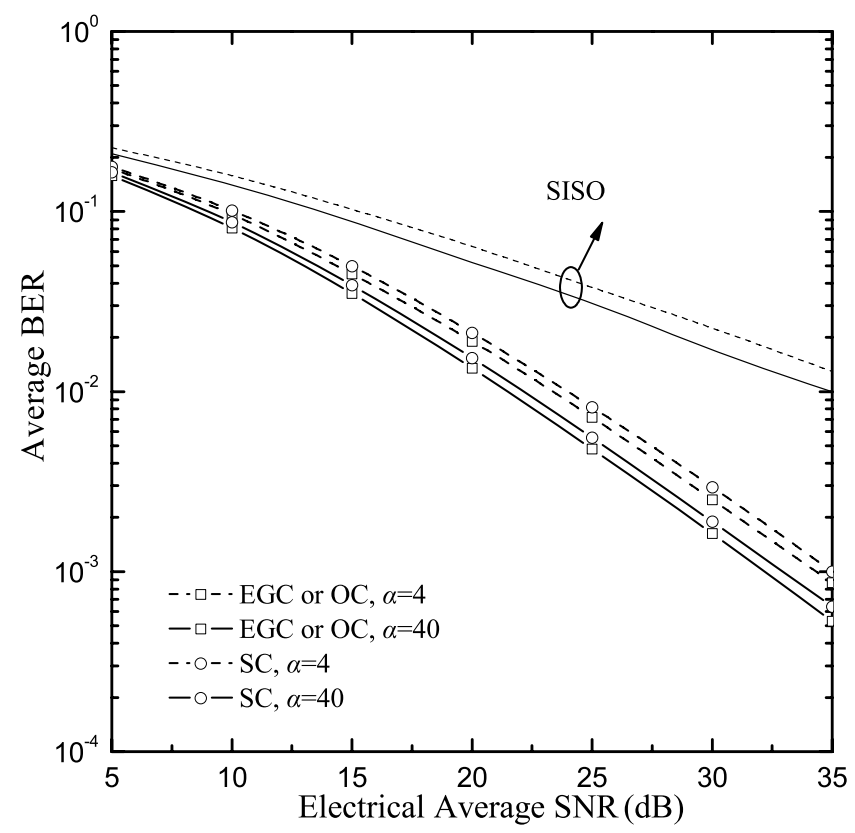

Fig. 4. Comparison of the $\mathrm{OC}$ and EGC receivers with $\mathrm{SC}$ receivers for SIMO FSO links for $N=2$ over i.i.d. turbulent channels.

performance with the deployment of a large receive aperture whose area is the same as the total area of smaller ones'. Note that Fig. 3 has been plotted using the approximation for the $Q$-function for OC and the GQR method for EGC.

Finally, in Fig. 4 the error performance of SIMO FSO links with $M=2$ receive apertures employing EGC, OC and SC over i.i.d. atmospheric turbulence channels, is depicted. As expected, it is shown that the performance of EGC/OC receivers outperforms SC ones. Therefore, the OC and EGC diversity schemes remain the most desired diversity schemes 
to mitigate error in uncoded optical wireless systems despite their circuitry complexity compared to SC receive aperture schemes.

\section{CONCLUSIONS}

In this paper, we have studied the error rate performance of FSO communication systems using spatial diversity over Kdistributed atmospheric turbulence channels. We have obtained accurate approximated closed-form expressions for the average BER of SIMO FSO systems in terms of Meijer G-function. Our results demonstrate that the use of multiple apertures at the transmitter and/or receiver enhance the quality of FSO systems similar to RF ones. In comparison to SISO case, a performance improvement of $110 \mathrm{~dB}$ is obtained at a target BER rate of $10^{-9}$ by using 3 transmit apertures. Moreover, it is shown that the required number of apertures over i.i.d. strong turbulence channels for transmit/receive diversity FSO systems in order to have a meaningful performance at a practical SNR value is more than 5 .

\section{REFERENCES}

[1] L. Andrews, R. L. Philips, and C. Y. Hopen, Laser Beam Scintillation with Applications. SPIE Press, 2001.

[2] D. Kedar and S. Arnon, "Urban optical wireless communications networks: The main challenges and possible solutions," IEEE Commun. Mag., vol. 42, no. 5, pp. 2-7, Feb. 2003.

[3] T. A. Tsiftsis, H. G. Sandalidis, G. K. Karagiannidis, and N. C. Sagias, "Multihop free-space optical communications over strong turbulence channels," in Proc. IEEE Int. Conf. Commun. (ICC'06), Istanbul, Turkey, June 2006.

[4] M. Safari and M. Uysal, "Relay-assisted free-space optical communication," in Proc. Asilomar Conf. Signals, Systems Computers, Invited Paper, Monteray, CA, USA, 2007.

[5] E. Jakeman and P. N. Pusey, "A model for non-Rayleigh sea echo," IEEE Trans. Antennas Propag., vol. 24, pp. 806-814, Nov. 1976.

[6] M. Uysal and J. T. Li, "BER performance of coded free-space optical links over strong turbulence channels," in Proc. IEEE Veh. Technol. Conf. (VTC spring), Milan, Italy, May 2004, pp. 168-172.

[7] M. Uysal, S. M. Navidpour, and J. T. Li, "Error rate performance of coded free-space optical links over strong turbulence channels," IEEE Commun. Lett., vol. 8, pp. 635-637, Oct. 2004.

[8] K. Kiasaleh, "Performance of coherent DPSK free-space optical communication systems in K-distributed turbulence," IEEE Trans. Commun., vol. 54, no. 4, pp. 604-607, Apr. 2006.

[9] X. Zhu and J. M. Kahn, "Performance bounds for coded free-space optical communications through atmospheric turbulence channels," IEEE Trans. Commun., vol. 51, no. 8, pp. 1233-1239, Aug. 2003.

[10] — "Markov chain model in maximum-likelihood sequence detectionfor free-space optical communication through atmospheric turbulence channels," IEEE Trans. Commun., vol. 51, no. 3, pp. 509-516, Mar. 2003.

[11] M. M. Ibrahim and A. M. Ibrahim, "Performance analysis of optical receivers with space diversity reception," in Proc. IEEE Commun., vol. 143, no. 6, pp. 369-372, Dec. 1996.

[12] E. J. Shin and V. W. S. Chan, "Optical communication over the turbulent atmospheric channel using spatial diversity," in Proc. IEEE Conf. Global Commun. (GLOBECOM'O2), Nov. 2002.

[13] — - "Part1: optical communication over the clear turbulent atmospheric channel using diversity," IEEE J. Select. Areas Commun., vol. 22, no. 9, pp. 1896-1906, Nov. 2004.

[14] S. G. Wilson, M. Brandt-Pearce, Q. Cao, and M. Baedke, "Optical repetition MIMO transmission with multipulse PPM," IEEE Trans. J. Select. Areas Commun., vol. 9, no. 23, pp. 1901-1910, Sept. 2005.

[15] S. G. Wilson, M. Brandt-Pearce, Q. Cao, and J. H. Leveque-III, "Free-space optical MIMO transmission with Q-ary PPM," IEEE Trans. Commun., vol. 53, no. 8, pp. 1402-1412, Aug. 2005.

[16] S. M. Navidpour, M. Uysal, and M. Kavehrad, "BER performance of free-space optical transmission with spatial diversity," IEEE Trans. Wireless Commun., vol. 6, no. 8, pp. 2813-2819, Aug. 2007.

[17] J. B. Carruthers and J. M. Kahn, "Angle diversity for nonidentical wireless infrared communication," IEEE Trans. Commun., vol. 48, no. 6, pp. 960-969, June 2000.
[18] A. G. Al-Ghambi and J. M. H. Elmirghani, "Analysis of diffuse optical wireless channels employing spot-diffusing techniques, diversity receivers, and combining schemes," IEEE Trans. Commun., vol. 52, no. 10 , pp. $1622-1631$, Oct. 2004

[19] I. S. Gradshteyn and I. M. Ryzhik, Table of Integrals, Series, and Products, 6th ed. New York: Academic, 2000.

[20] A. P. Prudnikov, Y. A. Brychkov, and O. I. Marichev, Integral and Series, vol. 3: More Special Functions. Amsterdam: Gordon and Breach Science Publishers, 1986.

[21] V. S. Adamchik and O. I. Marichev, "The algorithm for calculating integrals of hypergeometric type functions and its realization in REDUCE system," in Proc. Int. Conf. on Symbolic and Algebraic Computation, Tokyo, Japan, 1990, pp. 212-224.

[22] X. Zhu and J. M. Kahn, "Free-space optical communication through atmospheric turbulence channels," IEEE Trans. Commun., vol. 50, no. 8, pp. 1293-1300, Aug. 2002.

[23] M. Abramovitz and I. A. Stegun, Handbook of Mathematical Functions with Formulas, Graphs, and Mathematical Tables, 9th ed. New York: Dover, 1972.

[24] W. Gautschi, "Orthogonal polynomials and quadrature," Elec. Trans. Numer. Anal., vol. 9, pp. 65-76, 1999.

[25] G. H. Golumb and J. H. Welsh, "Calculation of Gauss quadrature rules," Math. Comput., vol. 23, pp. 221-230, Apr. 1969.

[26] M. Chiani, D. Dardari, and M. K. Simon, "New exponential bounds and approximations for the computation of error probability in fading channels," IEEE Trans. Wireless Commun., vol. 2, no. 4, pp. 840-845, July 2003.

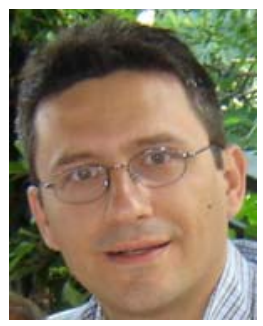

Theodoros A. Tsiftsis (S'02-M'04) was born in Lamia, Greece, in November 1970. He received the degree in Physics from the Aristotle University of Thessaloniki, Greece, in 1993, and the M.Sc. degree in Electrical Engineering from the HeriotWatt University, Edinburgh, Scotland, U.K., in 1995. Also, he received the M.Sc. degree in Decision Sciences from the Athens University of Economics and Business, Greece, in 2000 and the Ph.D. degree in Electrical Engineering from the University of Patras, Greece, in 2005. He is currently an Adjunct Assistant Professor in the Department of Informatics with Applications in Biomedicine, University of Central Greece, and he is also a visiting scientist at the Wireless Communications Systems Group (WCSG) in the Department of Electrical and Computer Engineering, Aristotle University of Thessaloniki, Greece.

His major research interests include cooperative communications, wireless communications theory, performance analysis over fading channels, and freespace optical communications.

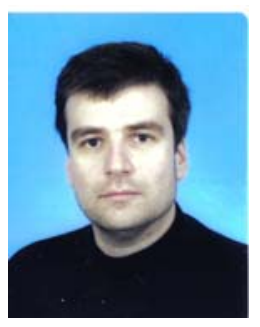

Harilaos G. Sandalidis was born in Florina, Greece, in 1972. He received the five year Diploma degree in Electronics and Computer Engineering and the MSc degree in Business Administration at the Production Engineering and Management Dept. from the Technical University of Crete, Greece in 1995 and 1998 respectively. He also received the MSc degree in Radiofrequency and Microwave Communications and the $\mathrm{PhD}$ degree in the Telecommunications area at the Electronics and Telecommunications (former Electronics and Electrical engineering) Dept. from the University of Bradford, UK, in 1996 and 2002 respectively

The period between 1996 and 2001 he was a research assistant at the Telecommunications Systems Institute of Crete, Greece working towards his $\mathrm{PhD}$ degree in collaboration with the Bradford University. After his military service he joined TEMAGON (former OTE Consulting) in 2002 where he worked as a telecom consultant for the risk mitigation program for the 2004 Olympic Telecommunication Network in collaboration with Telcordia Technologies, Inc. He is now working in the Greek Ombudsman as a senior investigator. He is also a Visiting Assistant Professor in the Dept. of Informatics with Applications in Biomedicine in the University of Central Greece. His major research interests include optical wireless communications, computational intelligence and heuristic optimization techniques regarding their application to the telecommunications field. 


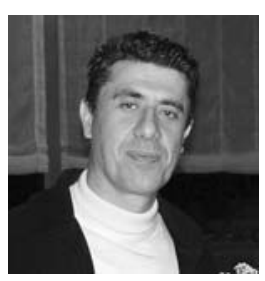

George K. Karagiannidis (M'97-SM'04) was born in Pithagorion, Samos Island, Greece. He received the University and Ph.D. degrees in electrical engineering from the University of Patras, Patras, Greece, in 1987 and 1999, respectively. From 2000 to 2004, he was a Senior Researcher at the Institute for Space Applications and Remote Sensing, National Observatory of Athens, Greece. In June 2004, he joined Aristotle University of Thessaloniki, Thessaloniki, Greece, where he is currently an Assistant Professor in the Electrical and Computer Engineering Department. His current research interests include wireless communication theory, digital communications over fading channels, cooperative diversity systems, cognitive radio, satellite communications, and wireless optical communications.

$\mathrm{He}$ is the author or coauthor of more than 80 technical papers published in scientific journals and presented at international conferences. He is also a coauthor of two chapters in books and a coauthor of the Greek edition of a book on mobile communications. He serves on the editorial board of the EURASIP JOURNAL ON WIRELESS COMMUNICATIONS AND NETWORKING

Dr. Karagiannidis has been a member of Technical Program Committees for several IEEE conferences. He is a member of the editorial boards of the IEEE TRANSACTIONS ON COMMUNICATIONS and the IEEE COMMUNICATIONS LetTers. He is co-recipient of the Best Paper Award of the Wireless Communications Symposium (WCS) in IEEE International Conference on Communications (ICC' 07), Glasgow, U.K., June 2007. He is a full member of Sigma Xi.

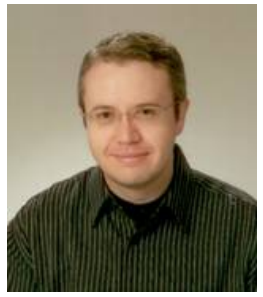

Murat Uysal was born in Istanbul, Turkey in 1973. $\mathrm{He}$ received the B.Sc. and the M.Sc. degree in electronics and communication engineering from Istanbul Technical University, Istanbul, Turkey, in 1995 and 1998, respectively, and the Ph.D. degree in electrical engineering from Texas A\&M University, College Station, Texas, in 2001. Since 2002, he has been with the Department of Electrical and Computer Engineering, University of Waterloo, Canada, where he is now an Associate Professor. His general research interests lie in communications theory and signal processing for communications with special emphasis on wireless applications. Specific research areas include space-time coding, MIMO techniques, cooperative communications, performance analysis over fading channels, and free-space optical communications.

Dr. Uysal is an Associate Editor for IEEE TRANS ACTIONS ON WIRELESS COMMUNICATIONS and IEEE COMMUNICATIONS LETTERS. He also served as a Guest Co-Editor for WILEY JOURNAL ON WiRELESS COMMUNICATIONS AND Mobile Computing's Special Issue on "MIMO Communications" published in 2004. Over the years, he has served on the technical program committee of more than 50 international conferences in the communications area. He co-chaired IEEE ICC (International Conference on Communications) Communication Theory Symposium in 2007 and chaired IEEE CCECE (Canadian Conference on Electrical and Computer Engineering) Communications and Networking Symposium in 2008. Dr. Uysal is a Senior IEEE member. 